\title{
EDITORIAL
}

\section{Rethinking the Exercise Electrocardiogram}

\author{
Paul Kligfield, M.D.
}

From the Department of Medicine, Division of Cardiology, Weill Medical College of Cornell University, NY

ECG changes during exercise-induced myocardial ischemia have been recognized for three quarters of a century, and exercise provocation of ST segment depression has been a widely used method for the diagnosis and assessment of coronary artery disease for well over 50 years. ${ }^{1,2}$ The exercise ECG is relatively inexpensive, widely accessible, and technically simple. In many circumstances, it can provide useful guidance regarding the presence, severity, and prognosis of coronary disease in symptomatic and asymptomatic patients with varying levels of pretest risk. However, it does so imperfectly. There are a number of problems that affect the utilization and interpretation of the test. Everyone has heard of someone who has had a myocardial infarction the week after a normal exercise test; what could be a more obvious indication of test fallibility? Some of these problems are likely unresolvable, while others may be solvable, but either way, they highlight a need to rethink the exercise ECG. Some of the following thoughts will find support in standard references, ${ }^{3-9}$ while others remain opinions. ${ }^{10-12}$

Demand-related myocardial ischemia is not a prerequisite for acute coronary events. It is catastrophic disruption of nonobstructive plaque that underlies the blood flow deficit in most forms of acute coronary syndrome. The traditional hypothesis that progressively obstructive coronary lesions cause progressively significant anginal symptoms, and then infarction, is true only occasionally. This cannot serve as a reasonable model for exercise test interpretation, and it is a limitation, but not a flaw, of the exercise ECG. Absence of functionally obstructive disease prior to the sentinel event is why approximately half of acute infarctions, often with sudden death, occur in patients with no prior angina. Accordingly, the normal exercise ECG found in these patients prior to the acute event in most cases is not a "false-negative" test, but rather an indication that severly obstructive coronary disease is not present in this subgroup. This limits the ability of the exercise ECG to predict plaque disruption, but it is not unique to the ECG: this important and perhaps insurmountable predictive limitation is shared by other imaging modalities as well. Of course, acute coronary syndromes also occur with plaque disruption in patients with preexisting extensive occlusive coronary disease, and this subgroup of patients should be identifiable, even when asymptomatic, by a more effective exercise ECG.

The exercise ECG is at a competitive disadvantage with newer diagnostic methods. This is a result of progress. Progress in imaging has been remarkable, and progress in pharmacology and in acute intervention has revolutionized the management of coronary disease, including stable angina and the acute coronary syndromes. But unlike ST elevation during infarction, ST segment depression on the exercise ECG does not localize myocardial ischemia in a way that allows useful prediction of the pattern or extent of coronary obstruction. It offers little insight into global ejection fraction, regional ventricular performance, or viable myocardium within areas of wall motion abnormality that might determine the course of acute intervention. Further, the ST segment response to exercise is not a particularly useful measure of the success of recent surgical or percutaneous revascularization, and its predictive value after acute infarction has been markedly reduced in the thrombolytic and interventional environment.

To remain viable, the exercise ECG must evolve beyond its widely perceived role as the diagnostic test that does not work very well. The value of exercise-induced ST depression is limited by poor

Address for reprints: Paul Kligfield, M.D., Weill Medical College of Cornell University, 525 East 68th Street, New York, NY 10021. Fax: 212-746-8561; E-mail: pkligfi@med.cornell.edu 
sensitivity, widely varying specificity, poor predictive value for the detection of coronary disease in populations with a low prevalence of coronary disease, and imperfect negative predictive value in higher risk patients. It performs even less well in women than in men, and its findings are inconclusive in patients with hypertension, valvular disease, and cardiomyopathy. It has only modest independent predictive value for future coronary events, particularly when added to an increasing number of nonexercise laboratory and demographic markers of cardiovascular risk. Diagnostic limitation of the standard exercise ECG has become an established principle of general medicine, which is only partially deserved. It has limitations, but many of these are shared by alternative diagnostic imaging, and many can be avoided by careful application of the test within specific populations.

Sensitivity of the exercise ECG need not be limited by empiric ST segment depression criteria that were established half a century ago. We can do better. This requires maximizing the information that can be extracted from the total ECG signal during recovery as well as during exercise, not just the end-exercise ST segment. Because ST depression is directly dependent on myocardial workload, it is unlikely that a fixed partition value such as $0.1 \mathrm{mV}$ of ST depression can serve as an optimal marker of the presence or extent of coronary artery disease. Reliance on a discrete amount of ST segment shift as a marker of ischemia, along with comparable prevalence of upsloping ST depression in normal subjects and patients with coronary disease, has been the critical limitations of the test. Poor test sensitivity has been the major diagnostic consequence, and while this can be altered along the well-trodden receiver-operating characteristic curve for ST segment depression, it can only be raised at the expense of specificity. Alternative ECG findings have the potential to increase both test sensitivity and specificity. Examples include heart rate correction of ST depression in exercise and in recovery, changes in QRS duration, amplitude-based QRS scores, and non-ST segment QT subinterval features. These newer methods require more widespread testing, alone and also in combination, for clarification of their values and limitations.

False-positive exercise ECG responses are not the major problem they used to be. One of the major difficulties with the exercise ECG has been the management of patients with "false-positive" tests.
The test specificity problem has been exaggerated by the inclusion of catheterized patients with chest pain syndromes but normal coronary arteries as "normals" in study populations. Many of these patients were referred for angiography because of an abnormal exercise ECG in the first place, often caused by noncoronary disorders such as hypertrophy and cardiomyopathy. Recent evidence indicates that test specificity in the general population is actually quite high. Even so, no test can escape the inevitable Bayesian reality that "falsepositive" tests can exceed "true-positive" tests when the pretest likelihood of disease is low. However, a strategy of serial testing with other imaging modalities can ease this problem.

Scores that incorporate multiple demographyand performance-based risk factors can improve the prognostic value of the exercise ECG. At issue here is whether these scores are about the exercise ECG or about risk factors and good clinical sense. It stands to reason that factors that increase the likelihood of underlying vascular pathology should improve the predictive value of the exercise test. The easiest and earliest example is maximal effort tolerance, which for many years has served to predict not only cardiovascular mortality but also cancer mortality as well. Incorporation of exercise duration along with a symptom grade reflecting angina severity adds to the predictive value of ST depression in the widely used Duke Jeopardy Score. Newer scores improve risk prediction by incorporating age, sex, diabetes, hypertension, smoking, and lipid levels with the ST segment response, and occasionally with peak heart rate achieved. Other predictive measures found during exercise testing, such as heart rate recovery, have value that is independent of the ECG response itself, perhaps as a global measure of overall parasympatheticsympathetic balance. These scores seem to work, and perhaps even more precisely than predictions assembled from the same data by excellent clinicians, but they are not really about the exercise ECG. Improved value of the ECG signal within multivariate scores should be our goal.

The exercise ECG should play a greater role in guiding the medical and interventional management of symptomatic patients with coronary artery disease. Patterns of practice in the management of patients with established coronary artery disease vary widely, but unless we arrive at a standard of care that mandates interventional revascularization for any and all obstructive coronary lesions, 
medical management of ischemic heart disease remains an appropriate option for stable patients at relatively low risk of adverse events. Exercise ECG positivity, alone, should not trigger placement of a drug-eluting stent without consideration of additional test factors, such as effort capacity and exercise workload. The exercise ECG can play an important role in estimating the overall anatomic, functional, and predictive risk in affected populations without confounding characteristics, as it did in earlier times in the selection of patients for medical versus open chest bypass surgery. This process can be aided by incorporation of some of the newer multivariate scoring systems in decision making.

Exercise testing of asymptomatic low-risk subjects should be reconsidered as the exercise ECG evolves. Guidelines currently recommend that, for the purpose of diagnosis of coronary disease, the exercise ECG be applied only in patients with "intermediate" probabilities of disease. Although widely defined as pretest disease likelihoods ranging from $10 \%$ to $90 \%$, the lower bound of intermediate probability effectively excludes large numbers of asymptomatic men and women without major risk factors from study. The argument against testing of low-risk subjects is based predominantly on the larger absolute increment in probability of disease that can be attributed to the test in the middle of the pretest likelihood spectrum than at either pretest tail. Indeed, for any test with imperfect test specificity, post-test positive predictive value (i.e., post-test likelihood of disease) can fall well below $10 \%$ in the lower $10 \%$ bound. But this recommendation might deserve review, based on several test developments. While absolute change in risk may be small, the relative increase of risk with a positive exercise ECG is actually highest in the low-risk group. Enhanced test specificity, and to a smaller extent enhanced test sensitivity, in selected low-risk asymptomatic populations can favorably alter the relationship between pretest and post-test likelihoods of disease. Methods are available to ascertain false-positive tests by serial study, and application of predictive scores can further clarify future risk. Identification of early or mild disease would more practically define a population who are likely to benefit from intensive prevention measures. Whether such measures would actually reduce adverse outcomes in subjects selected in this way remains to be established.

\section{REFERENCES}

1. Goldschlager N, Selzer A, Cohn K. Treadmill stress tests as indicators of presence and severity of coronary artery disease. Ann Intern Med 1976;85:277-286.

2. Chaitman BR. The changing role of the exercise electrocardiogram as a diagnostic and prognostic test for chronic ischemic heart disease. J Am Coll Cardiol 1986;8:1195-1210.

3. Detrano R, Salcedo E, Passalacqua M, et al. Exercise electrocardiographic variables: A critical appraisal. J Am Coll Cardiol 1986;8:836-847.

4. Gianrossi R, Detrano R, Mulvihill D, et al. Exercise-induced ST depression in the diagnosis of coronary artery disease. A meta-analysis. Circulation 1989;80:87-98.

5. Okin PM, Kligfield P. Heart rate adjustment of ST segment depression and performance of the exercise electrocardiogram: A critical evaluation. J Am Coll Cardiol 1995;25:1726-1735.

6. Froelicher VF, Lehmann KG, Thomas R, et al. The electrocardiographic exercise test in a population with reduced workup bias: Diagnostic performance, computerized interpretation, and multivariable prediction. Veterans Affairs Cooperative Study in Health Services \#016 (QUEXTA) Study Group. Quantitative Exercise Testing and Angiography. Ann Intern Med 1998;128:965-974.

7. Cole CR, Blackstone EH, Pashkow FJ, et al. Heart-rate recovery immediately after exercise as a predictor of mortality. N Engl J Med 1999;341:1351-1357.

8. Gibbons RJ, Balady GJ, Bricker JT, et al. ACC/AHA 2002 guideline update for exercise testing: Summary article: A report of the American College of Cardiology/American Heart Association Task Force on Practice Guidelines (Committee to Update the 1997 Exercise Testing Guidelines). Circulation 2002; 106:1883-1892.

9. Morise AP, Olson MB, Merz CN, et al. Validation of the accuracy of pretest and exercise test scores in women with a low prevalence of coronary disease: The NHLBI-sponsored Women's Ischemia Syndrome Evaluation (WISE) study. Am Heart J 2004;147:1085-1092.

10. Kligfield P, Okin PM. Evolution of the exercise electrocardiogram. Am J Cardiol 1994;73:1209-1210.

11. Kligfield P. Rehabilitation of the exercise electrocardiogram. Ann Intern Med 1998;128:1035-1037.

12. Lauer $M$, Froelicher ES, Williams $M$, et al. Exercise testing in asymptomatic adults: A statement for professionals from the American Heart Association Council on Clinical Cardiology, Subcommittee on Exercise, Cardiac Rehabilitation, and Prevention. Circulation 2005;112:771-776. 